\title{
Anticholinergic Cognitive Burden as a Predictive Factor for In-hospital Mortality in Older Patients in Korea
}

\author{
Jae Hyun Lee ${ }^{1,{ }^{*}}$, Hee-Won Jung ${ }^{1, *}$, Il-Young Jang ${ }^{2}$, Sung do Moon ${ }^{1}$, Sunhye Lee ${ }^{1}$, Seung Jun Han ${ }^{1}$ \\ ${ }^{1}$ Department of Internal Medicine, Seoul National University Hospital, Seoul, Korea \\ ${ }^{2}$ Division of Geriatrics, Department of Internal Medicine, Asan Medical Center, University of Ulsan College of Medicine, Seoul, Korea
}

Corresponding Author:

Seung Jun Han, MD

Department of Internal Medicine,

Seoul National University Hospital, 101

Daehak-ro, Jongno-gu, Seoul 03080,

Korea

E-mail: 82917@snuh.org

ORCID:

https://orcid.org/0000-0001-8960-3542

*These authors contributed equally to this work.

Received: December 11, 2019

Revised: January 31, 2020

Accepted: February 7, 2020
Background: With the increasing prevalence of chronic disease due to aging, many older adults experience multimorbidity and polypharmacy. Medications with anticholinergic properties are particularly associated with adverse cognitive outcomes, including functional decline and mortality. We assessed the clinical impact of anticholinergic cognitive burden (ACB) on clinical outcomes of older patients acutely admitted to a single, hospitalist-operated medical unit of a tertiary hospital in Korea. Methods: This retrospective study reviewed electronic medical records of 318 patients aged 65 years or older admitted to the hospitalist-operated medical unit through the emergency department of Seoul National University Hospital. The analyzed clinical outcomes were the length of hospital stay, in-hospital mortality, unplanned intensive care unit admission, and unexpected readmission within 30 days. Results: The clinical outcomes did not differ between patients who took five or more drugs and those who did not. Patients with an ACB score of 3 or higher had a higher in-hospital mortality rate and longer hospital stay than those who did not. After adjusting for confounding factors, an ACB score of 3 or higher was an independent predictive factor for in-hospital mortality (odds ratio=3.09; 95\% confidence interval, 1.18-8.06). Conclusion: ACB rather than the number of medications was associated with in-hospital mortality in acutely ill older patients. Further analytic and interventional studies are required to assess potentially inappropriate medication use and ACB in older inpatients.

Key Words: Geriatrics, Multimorbidity, Polypharmacy, Mortality, Length of stay

\section{INTRODUCTION}

With the increasing prevalence of chronic disease with aging, many older adults are treated concurrently for two or more diseases, a condition commonly referred to as a state of multimorbidity. ${ }^{1,2)} \mathrm{A}$ recent report indicated that the prevalence of multimorbidity in Korea is up to $73 \%{ }^{3)}$ predominantly due to common diseases such as hypertension, osteoarthritis, and hyperlipidemia. Since medical management for these conditions requires medications for specific diseases, older adults with multimorbidity are likely to take multiple medications simultaneously. Consequently, polypharmacy, a geriatric condition defined as taking multiple medications (usually five or more per day) is a frequently encountered clinical condition in medical care for older adults. ${ }^{4,5)}$

Medical care for older patients, especially those with polypharmacy, should consider factors such as prescribing cascade, drugdrug interactions, drug-disease interactions, and potentially inappropriate medications (PIMs) for older adults. ${ }^{6-9)}$ Among these factors, the presence of PIMs is reportedly associated with increased adverse outcomes, including delirium, falls, functional decline, and mortality. Therefore, guidelines have recommended to reduce the use of or to replace PIMs with safer alternatives. ${ }^{8-10)}$ Moreover, the concept of deprescribing, an individualized therapeutic strategy that considers the risks and benefits of medications according to patient functional and comorbid status, has emerged with efforts to minimize adverse outcomes with polypharmacy. ${ }^{11)}$ 
PIMs include a wide range of medications with anticholinergic properties affecting the cognitive states of older patients. ${ }^{8,12)}$ Clinical evidence has shown associations between these medications and adverse cognitive outcomes, ${ }^{13)}$ backed by evident scientific knowledge of the importance of acetylcholine signaling in cognitive performance. ${ }^{14,15)}$ Accordingly, varying clinical measures to quantify anticholinergic cognitive burden (ACB) have been developed and validated. ${ }^{13,16,17)}$

Older patients admitted through the emergency department in tertiary hospitals tend to have multimorbidity and polypharmacy, suggesting high exposure to anticholinergic medications in this population that may lead to adverse outcomes. ${ }^{18,19)}$ However, to our knowledge, no study has focused on ACB in acutely admitted older patients in Korea. Therefore, we assessed the clinical impact of ACB on clinical outcomes of older patients admitted via the emergency department of a single, hospitalist-operated medical unit of a tertiary hospital in Korea.

\section{MATERIALS AND METHODS}

\section{Clinical Setting and Study Design}

This retrospective study reviewed the electronic medical records at Seoul National University Hospital. We first searched for patients discharged from the hospitalist-operated medical unit at Seoul National University Hospital between February 2018 and October 2019. Among these patients, we included those 65 years of age and older admitted through the emergency department. To focus on acutely ill patients, we excluded patients admitted from the outpatient department and who were transferred from other wards, including the intensive care unit (ICU).

This study was carried out in accordance with the principles of the Declaration of Helsinki and was approved by the Institutional Review Board of Seoul National University Hospital (No. H-1911089-1079), which waived informed consent due to the retrospective nature of the study.

\section{Measurement of ACB}

Regular medications were assumed to be all the drugs regularly taken by each patient before hospitalization. We collected data on regular medications from each patient to check for polypharmacy and assessed the ACB by reviewing the patients' regular medications and those prescribed during hospitalization. Each patient's ACB score was calculated by summing the score according to the anticholinergic cognitive burden scale. ${ }^{17)}$

\section{Data Collections and Outcome Measures}

Demographic data such as sex and age and data on medical history were collected to calculate the Charlson Comorbidity Index (CCI). To assess the condition severity at the time of admission, vital signs and laboratory test results were also collected. Length of stay (LOS), in-hospital mortality, unplanned ICU admission, and unexpected readmission within 30 days were analyzed as clinical outcomes.

\section{Statistical Analysis}

Data are expressed as mean \pm standard deviation (SD) or numbers (percentage) unless stated otherwise. Chi-square or Fisher exact tests were used to compare categorical variables, while continuous variables were compared by Student t-tests. Univariate logistic regression was used to identify factors significantly influencing clinical outcomes. Multivariate logistic regression was performed with those factors to determine the independent predictive factors of ward mortality and delirium. Two-tailed p-values less than 0.05 were considered statistically significant. All statistical analyses were performed using IBM SPSS Statistics for Windows, version 23.0 (IBM Corp., Armonk, NY, USA).

\section{RESULTS}

\section{Baseline Characteristics and Clinical Outcomes in the Study Population}

This study included 318 patients. Among them, the mean age was $74.9 \pm 6.8$ years and 205 patients $(64.5 \%)$ were men. A total of 240 patients $(75.5 \%)$ were taking five or more drugs and the mean ACB score was 3.1 points. The proportions of patients with hypertension, diabetes, and malignancy were $47.5 \%, 38.7 \%$, and $71.1 \%$, respectively. Multimorbidity, defined as the co-existence of two or more chronic illnesses, was present in 208 patients (65.4\%) and mean the CCI was $7.5 \pm 2.6$ points. Regarding clinical outcomes, the in-hospital mortality and readmission rates within 30 days were $9.1 \%$ (29 patients) and $7.2 \%$ (23 patients), respectively. The mean LOS in the study population was $13.6 \pm 10.3$ days. Finally, 8 patients entered the ICU unexpectedly (Table 1).

\section{Comparisons of Patients according to the Number of Concurrent Regular Medications}

Patients taking five or more regular medications were categorized into the polypharmacy group. Age did not differ between the two groups. The proportion of men was higher in the polypharmacy group than that in the non-polypharmacy group (68.3 vs. 52.6\%; $\mathrm{p}=0.011$ ). Mean ACB and CCI score were higher in the polypharmacy group than those in the non-polypharmacy group $(3.2 \pm 2.7$ vs. $2.5 \pm 2.1$ and $7.7 \pm 2.6$ vs. $7.0 \pm 2$. ${ }^{6}$ respectively). More patients in the polypharmacy group had hypertension, diabetes, angina, and chronic kidney disease. Admission vital signs except for respi- 
Table 1. Study population baseline characteristics and clinical outcomes $(\mathrm{n}=318)$

\begin{tabular}{|c|c|}
\hline Variable & Value \\
\hline Age (y) & $74.9 \pm 6.8$ \\
\hline Men & $205(64.5)$ \\
\hline Polypharmacy & $240(75.5)$ \\
\hline Non-polypharmacy & $78(24.5)$ \\
\hline Number of regular medications & $8.4 \pm 5.0$ \\
\hline ACB score & $3.1 \pm 2.5$ \\
\hline CCI score & $7.5 \pm 2.6$ \\
\hline Multimorbidity & $208(65.4)$ \\
\hline \multicolumn{2}{|l|}{ Underlying illness } \\
\hline Hypertension & $151(47.5)$ \\
\hline Diabetes & $123(38.7)$ \\
\hline Malignancy & $226(71.1)$ \\
\hline Chronic lung disease & $38(11.9)$ \\
\hline History of myocardial infarction & $9(2.8)$ \\
\hline Heart failure & $31(9.7)$ \\
\hline Angina & $30(9.4)$ \\
\hline Asthma & $13(4.1)$ \\
\hline Arthritis & $30(9.4)$ \\
\hline Stroke & $25(7.9)$ \\
\hline Chronic kidney disease & $47(14.8)$ \\
\hline \multicolumn{2}{|l|}{ Vital signs upon admission } \\
\hline Systolic blood pressure (mmHg) & $126.0 \pm 22.0$ \\
\hline Diastolic blood pressure (mmHg) & $73.2 \pm 12.4$ \\
\hline Pulse rate (per minute) & $88.2 \pm 16.6$ \\
\hline Respiratory rate (per minute) & $21.4 \pm 3.2$ \\
\hline Body temperature $\left({ }^{\circ} \mathrm{C}\right)$ & $36.9 \pm 0.6$ \\
\hline \multicolumn{2}{|l|}{ Initial laboratory results upon admission } \\
\hline Hemoglobin (g/dL) & $10.3 \pm 2.3$ \\
\hline Serum albumin $(\mathrm{g} / \mathrm{dL})$ & $3.0 \pm 0.6$ \\
\hline Serum BUN (mg/dL) & $26.3 \pm 19.9$ \\
\hline Serum creatinine $(\mathrm{mg} / \mathrm{dL})$ & $1.3 \pm 1.3$ \\
\hline $\mathrm{eGFR}^{\mathrm{b})}\left(\mathrm{mL} / \mathrm{min} / 1.73 \mathrm{~m}^{2}\right)$ & $75.1 \pm 39.0$ \\
\hline Serum sodium $(\mathrm{mmol} / \mathrm{L})$ & $135.8 \pm 9.7$ \\
\hline \multicolumn{2}{|l|}{ Clinical outcomes } \\
\hline Length of stay (day) & $13.6 \pm 10.3$ \\
\hline In-hospital mortality & $29(9.1)$ \\
\hline Readmission within 30 days & $23(7.2)$ \\
\hline Unplanned ICU admission & $8(2.5)$ \\
\hline
\end{tabular}

Values are presented as mean \pm standard deviation or number (\%).

$\mathrm{ACB}$, anticholinergic cognitive burden; CCI, Charlson Comorbidity Index; BUN, blood urea nitrogen; eGFR, estimated glomerular filtration rate; ICU, intensive care unit.

${ }^{\text {a) }}$ Patients taking five or more concurrent regular medications.

${ }^{b}$ Based on the original Modification of Diet in Renal Disease (MDRD) equation.

ratory rate were similar between groups. Serum creatinine level on admission was higher in the polypharmacy group, although blood urea nitrogen (BUN) level and estimated glomerular filtration rate (eGFR) did not significantly differ between the two groups. No significant differences in clinical outcomes were observed between the two groups for in-hospital mortality, readmission rate, mean LOS, and unexpected ICU admission (Table 2).

\section{Comparisons of Patients according to ACB}

High ACB, defined as an ACB score of 3 or higher, was observed in 156 patients (49.1\%). Patients with high ACB scores were taking more concurrent regular medications than patients without high ACB scores (mean number of medications, $9.0 \pm 5.0$ vs. $7.8 \pm 5.0 ; \mathrm{p}=0.028$ ). Chronic kidney disease as an underlying illness was more common in patients with high ACB score. There were no differences in age, sex, $\mathrm{CCI}$ score, or admission vital signs between groups. Patients with high ACB score had lower serum albumin levels. Patients with an ACB score of 3 or higher also showed a higher in-hospital mortality rate (14.1 vs. $4.3 \%$; $\mathrm{p}=0.002$ ) and longer hospital stays (mean LOS, $16.2 \pm 11.6 \mathrm{vs}$. $11.2 \pm 8.2$ days; $\mathrm{p}<0.001$ ) than those who did not. No differences in the proportions of readmission within 30 days or unplanned ICU admissions were observed between groups (Table 3).

\section{Anticholinergic Burden as an Independent Predictive Factor for In-hospital Mortality}

Univariate regression analyses for all variables revealed factors such as $\mathrm{CCI}$ score, high $\mathrm{ACB}$, admission pulse rate, and serum albumin and BUN level on admission to be associated with in-hospital mortality. Age, sex, or five or more regular medications did not predict in-hospital mortality. After adjusting for confounding factors, an ACB score of 3 or higher remained an independent predictive factor for in-hospital mortality (odds ratio $[\mathrm{OR}]=3.09 ; 95 \%$ confidence interval $[\mathrm{CI}], 1.18-8.06)$. Moreover, one-point increment in CCI score ( $\mathrm{OR}=1.35 ; 95 \% \mathrm{CI}, 1.12-1.63)$, one beat per minute increment in pulse rate $(\mathrm{OR}=1.04 ; 95 \% \mathrm{CI}, 1.01-1.07)$, and $1-\mathrm{g} / \mathrm{dL}$ increment in serum albumin level $(\mathrm{OR}=0.36 ; 95 \% \mathrm{CI}$, 0.16-0.83) were also associated with in-hospital mortality (Table 4).

\section{DISCUSSION}

In this study, we found that older patients admitted via the emergency department had a high prevalence of polypharmacy and were also heavily exposed to medications with anticholinergic properties. Both univariate and multivariate analyses revealed that $\mathrm{ACB}$ and not polypharmacy, per se, was associated with in-hospital mortality. To our knowledge, this is the first study to report the association between ACB and in-hospital mortality in acutely ill patients in Korea. 
Table 2. Comparisons of patients according to the numbers of concurrent regular medications

\begin{tabular}{|c|c|c|c|}
\hline Variable & Polypharmacy $^{\mathrm{a})}(\mathrm{n}=240)$ & Non-polypharmacy $(\mathrm{n}=78)$ & p-value \\
\hline Age (y) & $75.0 \pm 6.6$ & $74.8 \pm 7.3$ & 0.798 \\
\hline Men & $164(68.3)$ & $41(52.6)$ & 0.011 \\
\hline ACB score & $3.2 \pm 2.7$ & $2.5 \pm 2.1$ & 0.037 \\
\hline CCI score & $7.7 \pm 2.6$ & $7.0 \pm 2.6$ & 0.037 \\
\hline \multicolumn{4}{|l|}{ Underlying illness } \\
\hline Hypertension & $123(51.3)$ & $28(35.9)$ & 0.018 \\
\hline Diabetes & $108(45.0)$ & $15(19.2)$ & $<0.001$ \\
\hline Malignancy & $168(70.0)$ & $58(74.4)$ & 0.461 \\
\hline Chronic lung disease & $31(12.9)$ & $7(9.0)$ & 0.351 \\
\hline History of myocardial infarction & $9(3.8)$ & 0 & 0.119 \\
\hline Heart failure & $27(11.3)$ & $4(5.1)$ & 0.129 \\
\hline Angina & $30(12.5)$ & 0 & 0.001 \\
\hline Asthma & $10(4.2)$ & $3(3.8)$ & $>0.999$ \\
\hline Arthritis & $26(10.8)$ & $4(5.1)$ & 0.181 \\
\hline Stroke & $21(8.8)$ & $4(5.1)$ & 0.467 \\
\hline Chronic kidney disease & $43(17.9)$ & $4(5.1)$ & 0.005 \\
\hline \multicolumn{4}{|l|}{ Vital signs upon admission } \\
\hline Systolic blood pressure (mmHg) & $127.1 \pm 22.7$ & $122.7 \pm 19.6$ & 0.123 \\
\hline Diastolic blood pressure (mmHg) & $73.5 \pm 12.3$ & $72.1 \pm 12.8$ & 0.380 \\
\hline Pulse rate (per minute) & $88.6 \pm 16.2$ & $86.6 \pm 17.8$ & 0.358 \\
\hline Respiratory rate (per minute) & $21.6 \pm 3.5$ & $20.8 \pm 2.0$ & 0.013 \\
\hline Body temperature $\left({ }^{\circ} \mathrm{C}\right)$ & $36.9 \pm 0.6$ & $36.9 \pm 0.7$ & 0.673 \\
\hline \multicolumn{4}{|l|}{ Initial laboratory results upon admission } \\
\hline Hemoglobin (g/dL) & $10.4 \pm 2.3$ & $10.3 \pm 2.4$ & 0.915 \\
\hline Serum albumin $(\mathrm{g} / \mathrm{dL})$ & $3.0 \pm 0.6$ & $3.0 \pm 0.6$ & 0.909 \\
\hline Serum BUN (mg/dL) & $27.6 \pm 20.3$ & $23.1 \pm 18.7$ & 0.107 \\
\hline Serum creatinine $(\mathrm{mg} / \mathrm{dL})$ & $1.4 \pm 1.4$ & $1.1 \pm 0.9$ & 0.017 \\
\hline $\mathrm{eGFR}^{\mathrm{b})}\left(\mathrm{mL} / \mathrm{min} / 1.73 \mathrm{~m}^{2}\right)$ & $73.0 \pm 40.3$ & $81.5 \pm 34.5$ & 0.097 \\
\hline Serum sodium (mmol/L) & $135.4 \pm 10.7$ & $137.3 \pm 5.6$ & 0.120 \\
\hline \multicolumn{4}{|l|}{ Clinical outcomes } \\
\hline Length of stay (day) & $13.5 \pm 9.3$ & $14.0 \pm 13.0$ & 0.734 \\
\hline In-hospital mortality & $24(10.0)$ & $5(6.4)$ & 0.497 \\
\hline Readmission within 30 days & $20(8.3)$ & $3(3.8)$ & 0.218 \\
\hline Unplanned ICU admission & $8(3.3)$ & $0(0)$ & 0.207 \\
\hline
\end{tabular}

Values are presented as mean \pm standard deviation or number $(\%)$.

ACB, anticholinergic cognitive burden; CCI, Charlson Comorbidity Index; BUN, blood urea nitrogen; eGFR, estimated glomerular filtration rate; ICU, intensive care unit.

${ }^{\text {a) }}$ Patients taking five or more concurrent regular medications.

${ }^{\mathrm{b}}$ Based on the original Modification of Diet in Renal Disease (MDRD) equation.

As the vast majority of commonly prescribed medications retain anticholinergic properties and also have biologic effects on cognitive performance, studies have evaluated the associations between anticholinergic exposure and clinical outcomes in older patients. Although long-term anticholinergic exposure and cognitive decline have been reported, controversies remain regarding the relevance of the short-term outcomes of ACB. Although a large-scale study showed an association between anticholinergic exposure and 2-year mortality, ${ }^{20)}$ studies on in-hospital mortality indicated no definite adverse effect of anticholinergic exposure. ${ }^{21,22)}$ In our study, ACB remained a significant predictor of mortality even after adjusting for comorbidity burden and polypharmacy.

Several possible mechanisms may explain the relationship between ACB and in-hospital mortality. Delirium, an important and 
Table 3. Comparisons of patients according to ACB scores

\begin{tabular}{|c|c|c|c|}
\hline Variable & High $\mathrm{ACB}^{\mathrm{a})}(\mathrm{n}=156)$ & Low ACB $(n=162)$ & $\mathrm{p}$-value \\
\hline Age (y) & $75.3 \pm 6.4$ & $74.5 \pm 7.2$ & 0.289 \\
\hline Men & $108(69.2)$ & $97(59.9)$ & 0.081 \\
\hline Number of regular medications & $9.0 \pm 5.0$ & $7.8 \pm 5.0$ & 0.028 \\
\hline CCI score & $7.8 \pm 2.6$ & $7.3 \pm 2.6$ & 0.091 \\
\hline \multicolumn{4}{|l|}{ Underlying illness } \\
\hline Hypertension & $74(47.4)$ & $77(47.5)$ & 0.986 \\
\hline Diabetes & $63(40.4)$ & $60(37.0)$ & 0.540 \\
\hline Malignancy & $109(69.9)$ & $117(72.2)$ & 0.644 \\
\hline Chronic lung disease & $17(10.9)$ & $21(13.0)$ & 0.570 \\
\hline History of myocardial infarction & $5(3.2)$ & $4(2.5)$ & 0.746 \\
\hline Heart failure & $20(12.8)$ & $11(6.8)$ & 0.070 \\
\hline Angina & $16(10.3)$ & $14(8.6)$ & 0.622 \\
\hline Asthma & $6(3.8)$ & $7(4.3)$ & 0.831 \\
\hline Arthritis & $13(8.3)$ & $17(10.5)$ & 0.510 \\
\hline Stroke & $15(9.6)$ & $10(6.2)$ & 0.254 \\
\hline Chronic kidney disease & $31(19.9)$ & $16(9.9)$ & 0.012 \\
\hline \multicolumn{4}{|l|}{ Vital signs upon admission } \\
\hline Systolic blood pressure (mmHg) & $126.0 \pm 21.8$ & $126.0 \pm 22.3$ & 0.982 \\
\hline Diastolic blood pressure (mmHg) & $72.5 \pm 12.1$ & $73.8 \pm 12.8$ & 0.350 \\
\hline Pulse rate (per minute) & $87.6 \pm 15.8$ & $88.6 \pm 17.3$ & 0.590 \\
\hline Respiratory rate (per minute) & $21.6 \pm 3.8$ & $21.3 \pm 2.4$ & 0.364 \\
\hline Body temperature $\left({ }^{\circ} \mathrm{C}\right)$ & $36.9 \pm 0.6$ & $36.9 \pm 0.6$ & 0.574 \\
\hline \multicolumn{4}{|l|}{ Initial laboratory results upon admission } \\
\hline Hemoglobin $(\mathrm{g} / \mathrm{dL})$ & $10.1 \pm 2.2$ & $10.6 \pm 2.4$ & 0.084 \\
\hline Serum albumin $(\mathrm{g} / \mathrm{dL})$ & $2.9 \pm 0.6$ & $3.1 \pm 0.6$ & 0.001 \\
\hline Serum BUN (mg/dL) & $27.6 \pm 21.7$ & $25.0 \pm 18.0$ & 0.252 \\
\hline Serum creatinine (mg/dL) & $1.4 \pm 1.3$ & $1.3 \pm 1.3$ & 0.532 \\
\hline $\mathrm{eGFR}^{\mathrm{b})}\left(\mathrm{mL} / \mathrm{min} / 1.73 \mathrm{~m}^{2}\right)$ & $74.8 \pm 43.4$ & $75.4 \pm 34.4$ & 0.900 \\
\hline Serum sodium $(\mathrm{mmol} / \mathrm{L})$ & $135.5 \pm 12.1$ & $136.1 \pm 6.7$ & 0.581 \\
\hline \multicolumn{4}{|l|}{ Clinical outcomes } \\
\hline Length of stay (day) & $16.2 \pm 11.6$ & $11.2 \pm 8.2$ & $<0.001$ \\
\hline In-hospital mortality & $22(14.1)$ & $7(4.3)$ & 0.002 \\
\hline Readmission within 30 days & $14(9.0)$ & $9(5.6)$ & 0.239 \\
\hline Unplanned ICU admission & $4(2.6)$ & $4(2.5)$ & $>0.999$ \\
\hline
\end{tabular}

Values are presented as mean \pm standard deviation or number $(\%)$.

$\mathrm{ACB}$, anticholinergic cognitive burden; CCI, Charlson Comorbidity Index; BUN, blood urea nitrogen; eGFR, estimated glomerular filtration rate; ICU, intensive care unit.

${ }^{\text {a) }}$ Patients with an ACB score of 3 or higher.

${ }^{b)}$ Based on the original Modification of Diet in Renal Disease (MDRD) equation.

Table 4. Predictive factors of in-hospital mortality

\begin{tabular}{|c|c|c|c|c|c|c|}
\hline \multirow[t]{2}{*}{ Variable } & \multicolumn{3}{|c|}{ Univariate analysis } & \multicolumn{3}{|c|}{ Multivariate analysis } \\
\hline & OR & $95 \% \mathrm{CI}$ & $\mathrm{p}$-value & OR & $95 \% \mathrm{CI}$ & p-value \\
\hline Age (1-year increment) & 1.03 & $0.98-1.09$ & 0.275 & 1.03 & $0.97-1.10$ & 0.373 \\
\hline Men & 1.82 & $0.75-4.40$ & 0.184 & 0.83 & $0.31-2.25$ & 0.717 \\
\hline Five or more regular medications & 1.62 & $0.60-4.41$ & 0.343 & 1.17 & $0.38-3.56$ & 0.788 \\
\hline ACB score of 3 or higher & 3.64 & $1.51-8.78$ & 0.004 & 3.09 & $1.18-8.06$ & 0.021 \\
\hline CCI (1-point increment) & 1.38 & $1.17-1.62$ & $<0.001$ & 1.35 & $1.12-1.63$ & 0.001 \\
\hline Pulse rate (increment by $1 \mathrm{bpm}$ ) & 1.03 & $1.01-1.06$ & 0.006 & 1.04 & $1.01-1.07$ & 0.006 \\
\hline Serum albumin (1-g/dL increment) & 0.24 & $0.11-0.51$ & $<0.001$ & 0.36 & $0.16-0.83$ & 0.018 \\
\hline BUN (1-mg/dL increment) & 1.02 & $1.00-1.03$ & 0.037 & 1.01 & $1.00-1.03$ & 0.118 \\
\hline
\end{tabular}

ACB, anticholinergic cognitive burden; CCI, Charlson Comorbidity Index; bpm, beats per minute; BUN, blood urea nitrogen; OR, odds ratio; CI, confidence interval. 
preventable geriatric condition in hospitalized older adults, might be a mediator, as shown in studies on anticholinergic exposure, delirium, and mortality. For instance, a study from an acute care hospital in Canada reported delirium severity to be associated with a clinical-rated anticholinergic score. ${ }^{23)}$ Another study from the United States including patients receiving palliative care showed a similar association between anticholinergic exposure according to an anticholinergic risk scale and delirium incidence. ${ }^{24)}$ Both shortterm and long-term time associations between delirium and mortality risk have been demonstrated. ${ }^{25,26)}$ Unfortunately, as a retrospective study, we did not include delirium as a study variable because medical record review may fail to capture hypoactive delirium, which is reportedly worse in terms of clinical outcome.

The population in the present study had a relatively higher prevalence of polypharmacy with substantial ACB compared to those in previous studies in other countries on older acute patients. ${ }^{22,27,28)}$ There may be several explanations for this difference. Firstly, the study population in the present study was inpatients admitted to an acute unit of a top-tier hospital in Korea, with a predictably high comorbidity burden. Secondly, the concepts of anticholinergic medications and PIMs are relatively unrecognized in Korea. Although Korea is experiencing an extreme pace of population aging, the concept of geriatric medicine is rarely taught in medical schools. ${ }^{29)}$ Thirdly, specialized or fragmented care for older multimorbid patients might contribute to the occurrence of prescribing cascades that often involve PIMs. ${ }^{30)}$ While our retrospective, descriptive study cannot address the contributions of these factors that may affect PIM and ACB in older patients with multimorbidities, our findings underscore the need for further research on the current nationwide status of medication usage in older adults.

As a retrospective observation performed by medical record review, our study has several limitations. Since our observations were based on the medical records of patients admitted to a single, hospitalist-run medical unit in a tertiary hospital, the characteristics of the patients in our study are not generalizable to the older population nationwide in Korea. Furthermore, our study lacks important geriatric baseline parameters including frailty, cognitive function, and daily functioning, and relevant outcome variables of ACB such as delirium and falls. Similarly, the functional outcomes of patients after discharge were unavailable in this study. Based on the results of this hypothesis-generating study, our upcoming prospective study with an interventional arm deprescribing PIMs and minimizing anticholinergic burden will provide better answers on the mediating mechanisms between ACB and clinical adverse outcomes.

In conclusion, $\mathrm{ACB}$ but not polypharmacy was associated with in-hospital mortality in acutely ill older patients. We hope that the results of this study lead to further analytic and interventional studies on PIMs and ACB in older inpatients.

\section{ACKNOWLEDGMENTS}

\section{CONFLICT OF INTEREST}

The researchers claim no conflicts of interest.

\section{AUTHOR CONTRIBUTIONS}

Conceptualization, HWJ; Data curation, SM, SL, SJH; Investigation JHL, HWJ, IYJ; Methodology. JHL, HWJ, IYJ, SJH; Project administration, HWJ; Supervision, SJH; Writing-original draft, JHL, HWJ; Writing, review \& Editing, SJH.

\section{REFERENCES}

1. Jung HW, Kim KI. Multimorbidity in older adults. J Korean Geriatr Soc 2014;18:65-71.

2. Marengoni A, Angleman S, Melis R, Mangialasche F, Karp A, Garmen A, et al. Aging with multimorbidity: a systematic review of the literature. Ageing Res Rev 2011;10:430-9.

3. Jang IY, Lee HY, Lee E. Geriatrics fact sheet in Korea 2018 from national statistics. Ann Geriatr Med Res 2019;23:50-3.

4. Kojima T. The need for actions against polypharmacy in older people with frailty. Ann Geriatr Med Res 2018;22:111-6.

5. Hajjar ER, Cafiero AC, Hanlon JT. Polypharmacy in elderly patients. Am J Geriatr Pharmacother 2007;5:345-51.

6. Rochon PA, Gurwitz JH. Optimising drug treatment for elderly people: the prescribing cascade. BMJ 1997;315:1096-9.

7. Rochon PA, Gurwitz JH. The prescribing cascade revisited. Lancet 2017;389:1778-80.

8. By the 2019 American Geriatrics Society Beers Criteria Update Expert Panel. American Geriatrics Society 2019 Updated AGS Beers Criteria for Potentially Inappropriate Medication use in older adults. J Am Geriatr Soc 2019;67:674-94.

9. Kim MY, Etherton-Beer C, Kim CB, Yoon JL, Ga H, Kim HC, et al. Development of a consensus list of potentially inappropriate medications for Korean older adults. Ann Geriatr Med Res 2018;22:121-9.

10. Gallagher P, O'Mahony D. STOPP (Screening Tool of Older Persons' potentially inappropriate Prescriptions): application to acutely ill elderly patients and comparison with Beers' criteria. Age Ageing 2008;37:673-9.

11. Scott IA, Hilmer SN, Reeve E, Potter K, Le Couteur D, Rigby D, et al. Reducing inappropriate polypharmacy: the process of deprescribing. JAMA Intern Med 2015; 175:827-34.

12. Boustani M, Campbell N, Munger S, Maidment I, Fox C. Impact 
of anticholinergics on the aging brain: a review and practical application. Aging Health 2008;4:311-20.

13. Salahudeen MS, Hilmer SN, Nishtala PS. Comparison of anticholinergic risk scales and associations with adverse health outcomes in older people. J Am Geriatr Soc 2015;63:85-90.

14. Feinberg M. The problems of anticholinergic adverse effects in older patients. Drugs Aging 1993;3:335-48.

15. Blokland A. Acetylcholine: a neurotransmitter for learning and memory? Brain Res Brain Res Rev 1995;21:285-300.

16. Duran CE, Azermai M, Vander Stichele RH. Systematic review of anticholinergic risk scales in older adults. Eur J Clin Pharmacol 2013;69:1485-96.

17. Campbell N, Maidment I, Fox C, Khan B, Boustani M. C46: The 2012 update to the anticholinergic cognitive burden scale. J Am Geriatr Soc 2013;61(S1):S142-3.

18. Hein C, Forgues A, Piau A, Sommet A, Vellas B, Nourhashemi F. Impact of polypharmacy on occurrence of delirium in elderly emergency patients. J Am Med Dir Assoc 2014;15:850.

19. Samaras N, Chevalley T, Samaras D, Gold G. Older patients in the emergency department: a review. Ann Emerg Med 2010; 56:261-9.

20. Fox C, Richardson K, Maidment ID, Savva GM, Ma tthews FE, Smithard D, et al. Anticholinergic medication use and cognitive impairment in the older population: the medical research council cognitive function and ageing study. J Am Geriatr Soc 2011; 59:1477-83

21. Dauphinot V, Faure R, Omrani S, Goutelle S, Bourguignon L, Krolak-Salmon P, et al. Exposure to anticholinergic and sedative drugs, risk of falls, and mortality: an elderly inpatient, multicenter cohort. J Clin Psychopharmacol 2014;34:565-70.

22. Kidd AC, Musonda P, Soiza RL, Butchart C, Lunt CJ, Pai Y, et al.
The relationship between total anticholinergic burden (ACB) and early in-patient hospital mortality and length of stay in the oldest old aged 90 years and over admitted with an acute illness. Arch Gerontol Geriatr 2014;59:155-61.

23. Han L, McCusker J, Cole M, Abrahamowicz M, Primeau F, Elie M. Use of medications with anticholinergic effect predicts clinical severity of delirium symptoms in older medical inpatients. Arch Intern Med 2001;161:1099-105.

24. Zimmerman KM, Salow M, Skarf LM, Kostas T, Paquin A, Simone MJ, et al. Increasing anticholinergic burden and delirium in palliative care inpatients. Palliat Med 2014;28:335-41.

25. van den Boogaard M, Peters SA, van der Hoeven JG, Dagnelie PC, Leffers P, Pickkers P, et al. The impact of delirium on the prediction of in-hospital mortality in intensive care patients. Crit Care 2010;14:R146.

26. van Hemert AM, van der Mast RC, Hengeveld MW, Vorstenbosch M. Excess mortality in general hospital patients with delirium: a 5-year follow-up of 519 patients seen in psychiatric consultation. J Psychosom Res 1994;38:339-46.

27. Corsonello A, Pedone C, Corica F, Incalzi RA. Polypharmacy in elderly patients at discharge from the acute care hospital. Ther Clin Risk Manag 2007;3:197-203.

28. Mizokami F, Koide Y, Noro T, Furuta K. Polypharmacy with common diseases in hospitalized elderly patients. Am J Geriatr Pharmacother 2012;10:123-8.

29. Jung HW, Lim JY. Geriatric medicine, an underrecognized solution of precision medicine for older adults in Korea. Ann Geriatr Med Res 2018;22:157-8.

30. Gill SS, Mamdani M, Naglie G, Streiner DL, Bronskill SE, Kopp A, et al. A prescribing cascade involving cholinesterase inhibitors and anticholinergic drugs. Arch Intern Med 2005;165:808-13. 